\title{
Novel Family of Chiral-Based Topological Insulators: Elemental Tellurium under Strain
}

\author{
Luis A. Agapito, ${ }^{1}$ Nicholas Kioussis, ${ }^{1, *}$ William A. Goddard III, ${ }^{2}$ and N. P. Ong ${ }^{3}$ \\ ${ }^{1}$ Department of Physics and Astronomy, California State University Northridge, Northridge, California 91330, USA \\ ${ }^{2}$ Division of Chemistry and Chemical Engineering, California Institute of Technology, Pasadena, California 91125, USA \\ ${ }^{3}$ Department of Physics, Princeton University, Princeton, New Jersey 08544, USA
}

(Received 5 November 2012; published 24 April 2013)

\begin{abstract}
Employing $a b$ initio electronic structure calculations, we predict that trigonal tellurium consisting of weakly interacting helical chains undergoes a trivial insulator to strong topological insulator (metal) transition under shear (hydrostatic or uniaxial) strain. The transition is demonstrated by examining the strain evolution of the band structure, the topological $\mathbb{Z}_{2}$ invariant and the concomitant band inversion. The underlying mechanism is the depopulation of the lone-pair orbitals associated with the valence band via proper strain engineering. Thus, Te becomes the prototype of a novel family of chiral-based three-dimensional topological insulators with important implications in spintronics, magneto-optics, and thermoelectrics.
\end{abstract}

DOI: 10.1103/PhysRevLett.110.176401

PACS numbers: 71.15.Ap, 72.25.Dc, 73.20.At, 73.43.Nq

The recent discovery of three-dimensional (3D) topological insulators (TIs) has sparked intense efforts in the search for novel materials that exhibit this new quantum-mechanical state of matter driven by strong spin-orbit coupling, which leads to the appearance of spin-momentum-locked topologically protected surface states with a Dirac-cone energy dispersion [1-3]. Ongoing research efforts focus primarily on the prototypical family of $\mathrm{Bi}_{2} \mathrm{Te}_{3}, \mathrm{Bi}_{2} \mathrm{Se}_{3}$, and $\mathrm{Sb}_{2} \mathrm{Te}_{3}$ 3D TIs, which exhibit a quintuple layered structure along the $c$ axis of the hexagonal lattice. The intra- and interlayer coupling within one quintuple layer is covalentlike while the interaction between two quintuple layers is much weaker, predominantly of the van der Waals type [3-5].

The heavier group-VI elements selenium and tellurium are ubiquitous in most of the recently discovered binary or ternary TIs and exhibit a wide variety of interesting properties under pressure. They undergo complex structural changes [6], exhibit semiconductor-to-metal transitions [7], have unusual melting curves [8], and some of their high-pressure phases are superconducting at low temperatures [6]. At ambient conditions, tellurium has a trigonal crystal structure (Te-I) with space group $\mathrm{D}_{3}^{4}$ consisting of weakly interacting infinite helical chains arranged in a hexagonal array, which spiral around axes parallel to $c$. The unit cell shown in Fig. 1(a) has three atoms at the positions $(u, 0,0),(0, u, 1 / 3)$, and $(\bar{u}, \bar{u}$, $2 / 3$ ) in units of the lattice vectors [9], where $u$ is the internal atomic position parameter. Each atom forms strong covalentlike intrachain bonds with its two nearest neighbors (NNs) and weak van der Waals-like interchain bonds with its four next NNs, with bond lengths of $r=$ $2.91 \AA$ and $R=3.43 \AA$, respectively. This unique feature is reflected in liquid-state studies of $\mathrm{Te}$ which showed that the chain structure is retained above the melting temperature [8].

In this Letter we predict that Te-I, a noncentrosymmetric material, becomes a strong TI or topological metal (TM) under application of shear or uniform and uniaxial strain, respectively. In most reported TIs to date, the band inversion and the gap closing occur near the time-reversal invariant momenta (TRIM) $(\vec{k} \equiv-\vec{k} \bmod \vec{G}$, where $\vec{G}$ is a reciprocal-lattice vector), where the frontier valence band (VB) and conduction band (CB) are doubly degenerate, as required by Kramer's theorem. In sharp contrast, the

(a)
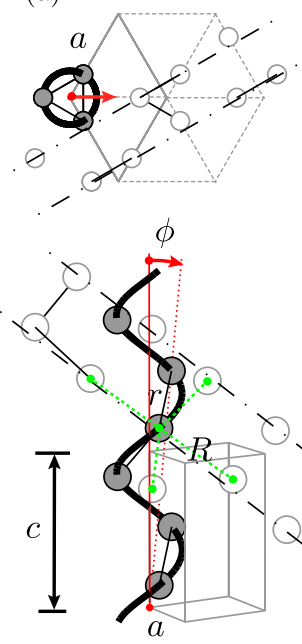

FIG. 1 (color online). (a) Three-dimensional and top views of trigonal Te-I consisting of right-handed helical chains arranged on a hexagonal lattice. Each atom has two intrachain NNs and four interchain next NNs with bond lengths $r$ and $R$, respectively. The dot-dashed lines show Kreb mesomeric chains. The shear strain tilts the axes of all chains with respect to the $c$ axis by an angle $\phi$ along the [110] direction [arrow in the top view panel (a)]. (b) Band structure of Te-I along the symmetry directions of the 3D BZ. The TRIM (non-TRIM) points are denoted by $\Gamma$ and $A$ ( $K$ and $H$ ). 
minimum band gap in Te-I occurs at a non-TRIM $(H)$, where the two frontier VBs are nondegenerate while the doubly degenerate $\mathrm{CB}$ is protected by the threefold screw symmetry of the helices. Thus, the transition to a TI state can be induced only by shear strain which breaks the threefold symmetry and lifts the twofold degeneracy of the CB state. We demonstrate the TI or TM nature by calculating the strain evolution of the $\mathbb{Z}_{2}$ topological invariants [10], which characterize the global band topology of the occupied Bloch wave functions in the entire Brillouin zone (BZ). This result is further corroborated by the evolution of the band structure and the concomitant band inversions between the frontier bands near $H$. We show that the underlying electronic mechanism is the interplay between the interchain lone pairs and the intrachain antibonding orbitals. Thus, Te becomes the prototype of a novel family of chiralbased 3D TIs of weakly interacting chains.

The ab initio electronic structure calculations employed the full-potential WIEN2K code [11] and the modified Becke-Johnson local-density approximation (MBJLDA) functional [12], which has been shown [13] to yield accurate band gaps, effective masses, and frontier-band ordering, which are crucial for the correct determination of the TI phase [14]. We have used the experimental unit cell parameters, $a=4.45 \AA$ and $c=5.93 \AA$. Atomic positions are relaxed using the Perdew-Burke-Ernzerhof functional [15]. For noncentrosymmetric systems, the $\mathbb{Z}_{2}$ can be determined either by integrating both the Berry's connection and curvature over half of the BZ [16], or equivalently, by calculating the evolution of the Wannier function center (WFC) in reciprocal space during a "time-reversal pumping" process $[10,17,18]$. This corresponds to the phase factor $\theta$ of the eigenvalues of the position operator $\hat{\mathbb{X}}$ projected into the occupied subspace. $\mathbb{Z}_{2}$ is then calculated by the even or odd number of crossings of the evolution of $\theta$ with any arbitrary horizontal $(\theta=$ const) reference line, $\bmod 2$. For a 3D TI, it is necessary to calculate the invariants $\left(\mathbb{Z}_{2}\right)_{0}$ and $\left(\mathbb{Z}_{2}\right)_{\pi}$ for the two different BZ planes $k_{3}=0$ and $k_{3}=G_{3} / 2$, where $G_{3}$ is the reciprocal-lattice vector. The system is a TI (trivial insulator) if the "strong" topological invariant $\nu_{0} \equiv\left[\left(\mathbb{Z}_{2}\right)_{\pi}-\left(\mathbb{Z}_{2}\right)_{0}\right](\bmod 2)$ is $1(0)$.

Zero strain.-The VB sextuplet ( $\sim-3$ to $0 \mathrm{eV})$ of Te at equilibrium shown in Fig. 1(b) arises from unhybridized $|p\rangle$ states, i.e., lone pairs. The bonding bands $(\sim-5$ to $-2.5 \mathrm{eV}$ ) arise from $\sigma$ hybridization of $|p\rangle$-like states, and the semicore bands $(\sim-14$ to $-8 \mathrm{eV})$ primarily from $|s\rangle$ states $[19,20]$. The $\mathrm{CB}$ sextuplet $(\sim 0$ to $\sim 3 \mathrm{eV})$ is primarily derived from antibonding $\sigma^{*}$-hybridized $|p\rangle$-like states. The calculated MBJLDA band gap of $0.21 \mathrm{eV}$ at the nonTRIM $H$ point $(1 / 3,1 / 3,1 / 2)$ is in good agreement with the experimental value of $0.33 \mathrm{eV}$ [21] and that of $0.32 \mathrm{eV}$ calculated with a screened-hybrid functional [22]. In addition, the MBJLDA yields dispersion and ordering of the frontier bands in excellent agreement with the hybrid method. We find that Te-I at equilibrium is a trivial insulator $\left(\nu_{0}=0\right)$ because the spin-orbit coupling is not large enough to cause inversion between frontier bands. Since Te does not exhibit crystal-inversion symmetry, Kramer's degeneracy is not required at the non-TRIM $H$ point. Nonetheless, because of the threefold screw symmetry of the helices, the CB state $H_{6}$ exhibits a twofold degeneracy [23], which is protected provided that the hexagonal lattice and the helical atomic ordering are preserved.

Finite strain.-For Te-I to become a TI, one needs to apply an external strain which can close the band gap, induce a crossing of the frontier bands (band inversion), and finally reopen the band gap. Interestingly, changes of the frontier VB and CB are associated with changes of $\nu_{0}(0 \leftrightarrow 1)$ and hence normal-insulator (NI) $\leftrightarrow$ TI or TM transitions [24]. We have investigated the evolution of $\nu_{0}$ upon the application of (i) hydrostatic compression, (ii) uniaxial strain parallel $\varepsilon_{\|}$and perpendicular $\varepsilon_{\perp}$ to $c$, with strain tensor matrix elements $\left(\epsilon_{1}=\epsilon_{2}=\epsilon_{4}=\epsilon_{5}=\right.$ $\epsilon_{6}=0$ and $\left.\epsilon_{3}=\varepsilon_{\|}\right)$and $\left(\epsilon_{1}=\varepsilon_{\perp}\right.$ and $\epsilon_{2}=\epsilon_{3}=\epsilon_{4}=$ $\epsilon_{5}=\epsilon_{6}=0$ ), respectively, and (iii) shear strain along the [110] direction in Fig. 1(a), where the primitive lattice vector $\vec{a}_{3}=c[\hat{z}+\hat{x}(\tan \phi)]$ and hence all helices are tilted by the angle $\phi$. We demonstrate that the transition to the TI phase requires breaking of the threefolded screw symmetry of the helices via shear strain.

Figure 2(a) shows schematically the evolution of the frontier VB (green) and CB (purple) in the vicinity of $H$ along $k_{z}$, from zero strain (central panel) to hydrostatic compression (right panel) and to shear strain (left panel). The change of band structure along the $H$ - $K$ direction

(a)
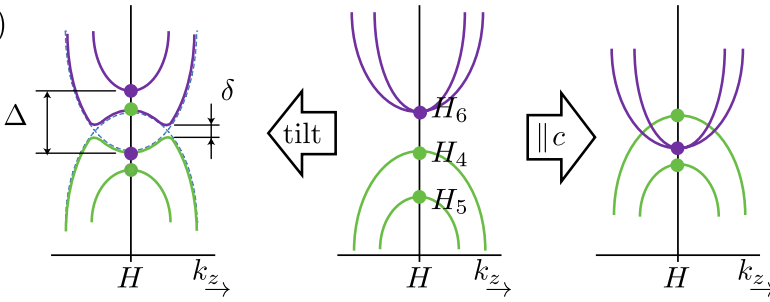

(b)

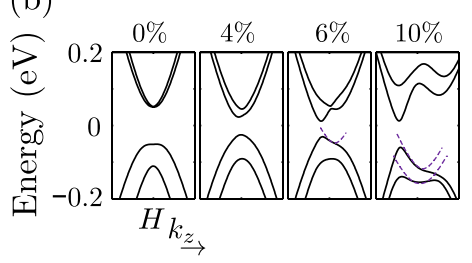

(c)

FIG. 2 (color online). Strain-induced crossing of the frontier bands along $k_{z}$ in the vicinity of $H$. (a) Left: Schematic bandinversion mechanism under shear strain where the twofold degeneracy of $H_{6}$ is lifted by $\Delta$ and the crystal-field splitting reopens a gap $\delta$ in the vicinity of $H$ after band crossing. Right: Under uniform (or uniaxial parallel to $c$ ) deformation the $H_{6}$ degeneracy is preserved after band inversion. Evolution of the MBJLDA frontier bands under (b) shear strain and under (c) hydrostatic pressure, in kbars. 
under application of shear strain $\left(\phi / 90^{\circ} \leq 10 \%\right)$ and hydrostatic compression $(P<39$ kbar $)$ are shown in Figs. 2(b) and 2(c), respectively, where we have used the experimental values of $a, c$, and $u$ for the latter case [9]. Hydrostatic compression preserves the threefold symmetry of the helices and thus the twofold degeneracy of the $H_{6}$ CB states. It shifts the VB and CB closing the band gap at $\sim 16$ kbar. Interestingly, we predict that band inversion occurs at this critical pressure and Te-I undergoes a transition to a topologically metal $\left(\nu_{0}=1\right)$ phase. For $P>$ $16 \mathrm{kbar}$, the gap does not reopen due to the fact that the trigonal crystal symmetry hinders hybridization between the $H_{4}$-VB and $H_{6}$-CB states.

Shear strain lifts the degeneracy of $H_{6}$ and reduces the energy gap in the vicinity of $H$ (hereafter denoted by $\tilde{H}$ ). The strain variation of the $\tilde{H}$ energy gap shown in Fig. 4(c) (black) indicates that it decreases from $0 \%-4.5 \%$ reaching the minimum at $\sim 4.5 \%-6 \%$ and then it increases with larger strain. In contrast to the uniform compression, the removal of the screw symmetry allows hybridization between the VB and $\mathrm{CB}$ which in turn prevents the closing of the gap. At $\approx 5.5 \%$ strain, the VB undergoes a sign reversal of the concavity [dashed line in the $6 \%$ panel of Fig. 2(b)] signaling the onset of band inversion between the $\mathrm{CB}$ and VB with reopening of a gap $\delta$. This in turn leads to the formation of a camel-back-shape (CBS) VB and an inverse CBS CB, and the transition to the TI phase $\left(\nu_{0}=1\right)$. At $\gtrsim 9 \%$ strain there is another sign reversal of the concavity of the second frontier VB which becomes positive (lower dashed line in the $10 \%$ panel) giving rise to the formation of two CBS VBs and two inverse CBS CBs, which connotes a four-band overlap analogous to the twoband-overlap sketch in Fig. 2(a). This leads to a second topological transition to the topologically trivial phase, where $\nu_{0}$ undergoes a $1 \rightarrow 0$ change.

Changes of the topological invariant.-Figures 3(a) and 3(b) show the evolution of the WFC along a complete period $\left(0 \leq k_{2} \leq\left(G_{2} / 2\right)\right)$ of the "time-reversal pumping" process [25] on the $k_{3}=G_{3} / 2 \mathrm{BZ}$ plane under (a) zero and (b) $6 \%$ shear strain, respectively. By projecting the

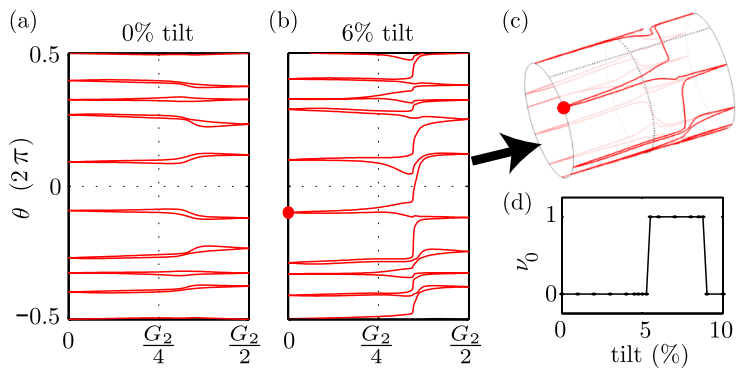

FIG. 3 (color online). Evolution of the WFC $\theta / 2 \pi$ along $0 \leq$ $k_{2} \leq\left(G_{2} / 2\right)$ on the $k_{3}=G_{3} / 2$ plane under (a) zero and (b) $6 \%$ shear strain, yielding a NI and a TI, respectively. (c) The Fu-Kane cylinder merges the periodic top and bottom edges of panel (b). (d) Evolution of $\nu_{0}$ with shear strain. evolution of the WFC on the Fu-Kane cylinder [25], one can see in Fig. 3(c) that under the $6 \%$ shear strain the added contributions of all the phases $\theta$ encloses the cylinder once, corresponding to an odd $(n=1)$ winding number. Thus, the $k_{3}=G_{3} / 2$ plane acquires a nontrivial timereversal polarization $P_{\theta} \equiv(2 \pi n / 2 \pi)=1$, or equivalently $\left(\mathbb{Z}_{2}\right)_{\pi} \equiv P_{\theta}=1[10,25]$. In contrast, under zero strain the phase $\theta$ in Fig. 3(a) does not continuously enclose the cylinder, corresponding to an even $(n=0)$ winding number and $\left(\mathbb{Z}_{2}\right)_{\pi}=0$. For all cases, $\left(\mathbb{Z}_{2}\right)_{0}=0$; i.e., the $k_{3}=0$ plane exhibits a trivial invariant, rendering the $6 \%$-strained (-unstrained) Te a topological (normal or trivial) insulator, where $\nu_{0}=1\left(\nu_{0}=0\right)$. Figure 3(c) displays the evolution of $\nu_{0}$ with shear strain, demonstrating that Te undergoes a $\mathrm{NI} \rightarrow$ TI phase transition at $\approx 5.5 \%$ followed by a second transition from topological to trivial at $\approx 9 \%$, consistent with the corresponding first and second band inversions in Fig. 2(b).

Electronic mechanism.-To elucidate the underlying mechanism of the transition to the TI phase, we show in Fig. 4(a) the bonding structure of a Te atom in the crystal occupying the center of a distorted octahedron with its six neighbors on multiple helices, where $\left\{r_{i}\right\}$ and $\left\{R_{i}\right\}$ denote $\mathrm{NN}$ intrachain and next NN interchain bond lengths. The octahedral basal plane is spanned by $r_{1}, r_{2}, R_{2}, R_{3}$, and the perpendicular $R_{3}-R_{4}$ axis aligns along a mesomeric chain [26]. The equilibrium intrachain $\vartheta$ and interchain $\gamma$ angles are $101^{\circ}$ and $167.5^{\circ}$, respectively. The $|h\rangle$ orbitals, which are $s p$ hybrids of mainly $|p\rangle$ character, form the intrachain covalentlike $\sigma$ bonds, while the $|\tau\rangle$ lone-pair orbitals, which are essentially unhybridized $|p\rangle$ states, form the
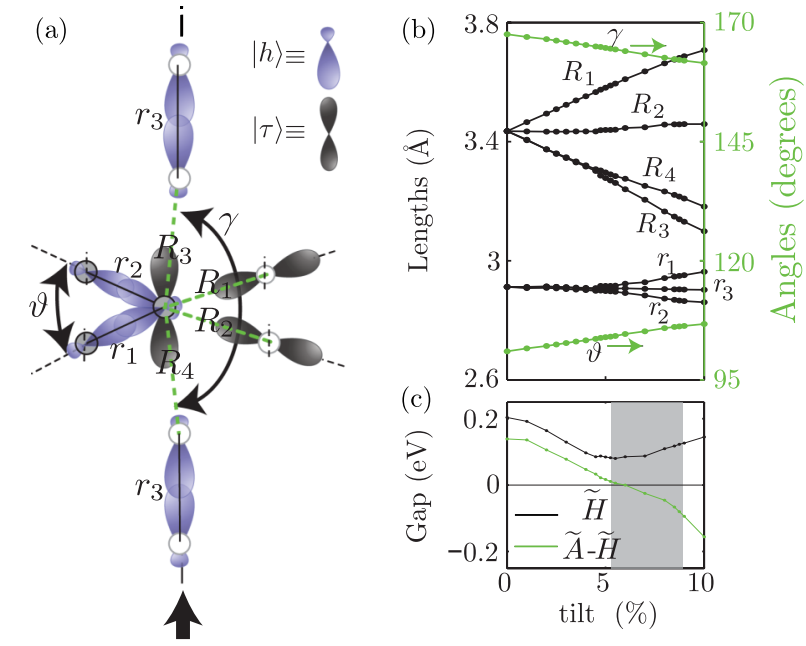

FIG. 4 (color online). (a) Schematic of bonding orbitals along the mesomeric chain $\ldots-r_{3}-R_{4}-R_{3}-r_{3}-R_{4}-R_{3}-\ldots$ shown in Fig. 1(a). $\left\{r_{i}\right\}$ and $\left\{R_{i}\right\}$ denote NN and next-NN bond lengths, respectively. The $|h\rangle$ orbitals are $s p$ hybrids while the lone pairs $|\tau\rangle$ are unhybridized $|p\rangle$ states [27]. (b) Variation of bond lengths and angles with shear strain. (c) Evolution of the "direct" $\tilde{H}$ and "indirect" $\tilde{A}-\tilde{H}$ energy gap as a function of tilt strain. The shaded area denotes the topological regime. 
interchain bonds [27]. Although only weakly coupled, these mesomeric chains knit together atoms from different helices and help stabilize the crystal through two mesomeric mechanisms [26] as follows. (1) Interactions between occupied orbitals: (a) The $|\tau\rangle-|\rho\rangle$ interaction, where $|\rho\rangle$ denotes the $|s\rangle$-derived semicore states [ $\sim-12 \mathrm{eV}$ in Fig. 1(b)], and (b) the interaction of $|\tau\rangle$ with the small back lobe of $|h\rangle$ along $R_{3}$ and $R_{4}$ in Fig. 4(a), forming secondary bonds at a slight expense to the primary bonds that also involve $|h\rangle$. (2) Interactions between unoccupied antibonding orbitals (not shown) and occupied $\tau$ orbitals: They do not involve the $\sigma$ bonding states and hence do not directly affect the primary bonds. This lonepair-antibonding hybridization $n p^{2} \rightarrow n \sigma^{*}$, often encountered in chalcogen chemistry [28], explains the closer proximity of the two lowest antibonding bands to the lone-pair bands rather than to the other bands of the antibonding sextuplet at $\tilde{H}$.

Shear strain.-Figure 4(b) shows the variation of the intrachain and interchain bond lengths and angles with shear strain. Increasing the tilt of the helices decreases the bond lengths $R_{3,4}$, hence strengthening these bonds in the mesomeric chains via the mechanism Fig. 1(b). It involves electron transfer from occupied lone-pair orbitals $|\tau\rangle$ to unoccupied antibonding states localized along $R_{3,4}$, which is corroborated by the strain evolution of the spatial distribution of the wave functions at $H$. On the other hand, the shear strain has a weaker effect on the intrachain bonds whose lengths $r_{1-3}$ remain almost constant for $\leqslant 5 \%$ strain. In addition, the depopulation of the lone-pair orbitals weakens the other interchain bonds whose bond lengths $R_{1}$ and $R_{2}$ elongate with increasing strain. At the critical strain of the NI $\rightarrow$ TI transition, the lone-pair orbital loses completely its electron; the concomitant filling of the antibonding orbital implies CB-VB band inversion at $\tilde{H}$. In other words, mechanical tilt of the helices induces "nucleation of dislocation lines" whose bonds are formed from previously inactive lone-pair electrons transferred into antibonding orbitals localized along these dislocation (mesomeric) lines.

Uniaxial strain.-Under a uniaxial compressive strain $\varepsilon_{\|}<0$ (both at constant volume and constant $a$ ), the band gap at $H$ increases. On the other hand, under a uniaxial tensile strain $\varepsilon_{\|}>0$, elongation of the helices increases the NN intrachain bond lengths $\left\{r_{i}\right\}$, resulting in a weakening of the $\sigma$ and $\sigma^{*}$ hybridization of the $|h\rangle$ orbitals [Fig. 4(a)] that reduces the energy splitting of the corresponding bonding and antibonding bands. Thus, tensile strain lowers the CB minimum (antibonding) at $\tilde{H}$ relative to the lonepair VB maximum, where the latter is less sensitive to such strain, thus leading to closing of the gap and band inversion. Interestingly, the effect of hydrostatic pressure is analogous to that of uniaxial tensile strain, where the gap closes with increasing pressure [21], because Te-I has a negative compression modulus along $c$ [9]. At a critical pressure $(P \approx 16$ kbar) the frontier bands in Fig. $2(\mathrm{c})$ cross, indicating a transition into the TM phase. Similar to shear strain, a second topological transition occurs at a higher pressure $(P>39 \mathrm{kbar})$ due to a second band crossing, which brings Te to a trivial metallic phase. Both topological transitions are corroborated by corresponding changes of $\nu_{0}$.

Application of a uniaxial transverse $\varepsilon_{\perp}$ tensile (compressive) strain increases (decreases) the separation between $\mathrm{NN}$ helices leading to the opening (closing) of the gap at $\tilde{H}$. In contrast to $\varepsilon_{\|}>0, \varepsilon_{\perp}<0$ reduces primarily the indirect $\tilde{A}-\tilde{H}$ gap over the $\tilde{H}$ gap. At $\sim-3.5 \%$ the indirect gap is closed and the system is rendered semimetallic, while the $\tilde{H}$ gap remains relatively large at $\sim 120 \mathrm{meV}$.

These calculations reveal simple guiding rules to tune the frontier bands and gap-closing process in this family of chiral-based 3D TIs under different types of deformations: (i) Uniaxial tensile $\varepsilon_{\|}>0$ and uniaxial compressive $\varepsilon_{\perp}<0$ strain controls the intrachain bond lengths $r$ and bond angles $\vartheta$, respectively, and hence has a primary effect on the covalentlike antibonding CB at $\tilde{H}$ [29]. (ii) Shear (tilt) strain controls the interchain lone-pair orbitals and has an effect primarily on the VB at $\tilde{H}$. (iii) Shear strain removes the threefold screw symmetry and hence activates the VB-CB hybridization (absent under both hydrostatic and uniaxial deformation) which allows the reopening of a $\delta$ gap at $\tilde{H}$. Shear strain defers the onset of semimetallicity (closing of the indirect $\tilde{A}-\tilde{H}$ gap) until after the system has transitioned into the topological insulating phase shown in Fig. 4(c) (green curve). Thus, shear seems to be the most effective strain mechanism to induce the NI $\rightarrow$ TI phase transition in Te.

In summary, $a b$ initio calculations demonstrate that Te-I undergoes a $\mathrm{NI} \rightarrow \mathrm{TI}(\mathrm{TM})$ transition under shear strain (hydrostatic compression or uniaxial tensile strain). We elucidate that the underlying mechanism is the depopulation of the lone-pair orbitals associated with the VB. This in turn leads to band inversion and the concomitant change of $\nu_{0}$. Understanding the rich chemistry of the lone pairs in chiral solids may have important implications for the discovery of the next-generation TI-based materials for spintronics, magneto-optics, and high-ZT thermoelectrics, such as the following. (i) Chirality-induced spin selectivity, where spin-polarized electrons have different tunneling properties in right- and left-handed helices depending on the electron spin polarization relative to the direction of sense advance of the helix [30]. This can be affected by the onset of gapless spin-textured surface states at the TI transition. (ii) A pair of protected 1D electron modes bound to the dislocation and propagating in opposite directions if the Burgers vector $\vec{b}$ of the dislocation satisfies the expression $\vec{b} \cdot \vec{k}=\pi \bmod (2 \pi)$, where $\vec{k}$ is a TRIM. [31] These protected 1D modes may provide a novel route to fabricating ideal Te quantum wires. The surface density of states of these 1D modes, which are absent in a nonchiral TI, will lead to an enhancement of the tunneling current 
measured by STM. (iii) A TI effect on the large dichroism that Te exhibits in the infrared, where the absorption constant depends on the polarization of the incident radiation in directions parallel and perpendicular to the $c$ axis.

We thank A. Soluyanov, E. Prodan, and T. Fukui for insightful discussions on the development of $\mathbb{Z}_{2}$ codes. The work at CSUN was supported by the NSF-PREM Grant No. DMR-1205734.

*nick.kioussis@csun.edu

[1] M.Z. Hasan and C. L. Kane, Rev. Mod. Phys. 82, 3045 (2010).

[2] L. Fu and C. L. Kane, Phys. Rev. B 76, 045302 (2007).

[3] H. Zhang, C.-X. Liu, X.-L. Qi, X. Dai, Z. Fang, and S.-C. Zhang, Nat. Phys. 5, 438 (2009).

[4] Y. Xia et al., Nat. Phys. 5, 398 (2009).

[5] Y. L. Chen et al., Science 325, 178 (2009).

[6] G. Parthasarathy and W. B. Holzapfel, Phys. Rev. B 37, 8499 (1988).

[7] P. W. Bridgman, Proc. Am. Acad. Arts Sci. 81, 165 (1952).

[8] B. C. Deaton and F. A. Blum, Jr., Phys. Rev. 137, A1131 (1965).

[9] R. Keller, W. B. Holzapfel, and H. Schulz, Phys. Rev. B 16, 4404 (1977).

[10] R. Yu, X. L. Qi, A. Bernevig, Z. Fang, and X. Dai, Phys. Rev. B 84, 075119 (2011).

[11] P. Blaha et al., "WIEN2k" Institute of Physical and Theoretical Chemistry, Vienna University of Technology, 2001.

[12] F. Tran and P. Blaha, Phys. Rev. Lett. 102, 226401 (2009).

[13] Y. S. Kim, M. Marsman, G. Kresse, F. Tran, and P. Blaha, Phys. Rev. B 82, 205212 (2010).
[14] J. Vidal, X. Zhang, L. Yu, J. W. Luo, and A. Zunger, Phys. Rev. B 84, 041109 (2011).

[15] J. P. Perdew, K. Burke, and M. Ernzerhof, Phys. Rev. Lett. 80, 891 (1998).

[16] E. Prodan, Phys. Rev. B 83, 235115 (2011); T. Fukui and Y. Hatsugai, J. Phys. Soc. Jpn. 76, 053702 (2007).

[17] A. A. Soluyanov and D. Vanderbilt, Phys. Rev. B 83, 235401 (2011).

[18] L. Winterfeld, L. A. Agapito, J. Li, N. Kioussis, P. Blaha, and Y.P. Chen, Phys. Rev. B 87, 075143 (2013).

[19] M. Kastner, Phys. Rev. Lett. 28, 355 (1972).

[20] F. Kirchhoff, N. Binggeli, G. Galli, and S. Massidda, Phys. Rev. B 50, 9063 (1994).

[21] V.B. Anzin, M. I. Eremets, Yu. V. Kosichkin, A.I. Nadezhdinskii, and A. M. Shirokov, Phys. Status Solidi A 42, 385 (1977).

[22] J. Heyd, G. E. Scuseria, and M. Ernzerhof, J. Chem. Phys. 118, 8207 (2003).

[23] R. Enderlein and A. Hache, Phys. Status Solidi B 60, 739 (1973).

[24] S. Murakami, Physica (Amsterdam) 43, 748 (2011).

[25] L. Fu and C. L. Kane, Phys. Rev. B 74, 195312 (2006).

[26] H. Krebs, Angew. Chem. 70, 615 (1958).

[27] R. M. Martin, G. Lucovsky, and K. Helliwell, Phys. Rev. B 13, 1383 (1976).

[28] I. Krossing, Handbook of Chalcogen Chemistry: New Perspectives in Sulfur, Selenium and Tellurium, edited by F. Devillanova (The Royal Society of Chemistry, London, 2007), p. 384.

[29] W. Lingelbach and G. Weiser, Phys. Status Solidi B 70, 205 (1975).

[30] S. Yeganeh, M. A. Ratner, E. Medina, and V. Mujica, J. Chem. Phys. 131, 014707 (2009).

[31] Y. Ran, Y. Zhang, and A. Vishwanath, Nat. Phys. 5, 298 (2009). 